\title{
Lange Überlebenszeit von Patienten bei malignem Melanom im Stadium IV ${ }^{1}$
}

\author{
Long-Term Survivors With Malignant Melanoma Stage IV
}

\author{
Autoren \\ H. Mohme, A. Tentrup, B. Heindl, J. Böttjer, R. Stadler \\ Institut \\ Hautklinik, Johannes Wesling Klinikum Minden
}

\section{Bibliografie}

Dol $10.1055 / \mathrm{s}-0028-1119648$

Akt Dermatol 2009; 35:

129-132 @ Georg Thieme

Verlag KG Stuttgart · New York

ISSN 0340-2541

Korrespondenzadresse

Prof. Dr. Rudolf Stadler

Direktor der Hautklinik

Johannes Wesling Klinikum

Hans-Nolte-Straße 1

32429 Minden

hautklinik@klinikum-minden.de

\section{Zusammenfassung \\ $\nabla$}

Das maligne Melanom ist ein hochmaligner melanozytärer Tumor, der im fern metastasierten Stadium IV einen fast immer letalen Ausgang nach kurzer Überlebenszeit hat. Wir präsentieren hier 11 Patienten, die von uns langfristig betreut wurden, deren Überlebenszeit bei Weitem länger war als diejenige, die von der AJCC 2001 angege-

\section{Einleitung \\ $\nabla$}

Das maligne Melanom ist ein hochmaligner melanozytärer Tumor. Für die Prognose sind die Tumordicke sowie das Vorhandensein von Lymphknotenmetastasen bzw. Fernmetastasen entscheidend. Die Metastasierung erfolgt zu 70\% primär lymphogen, zu $30 \%$ primär hämatogen [1]. Häufig kommt es zunächst zur Ausbildung von Satellitenmetastasen, dann zu In-TransitMetastasen und schließlich zum Befall regionaler Lymphknoten. Fernmetastasen können lymphogen oder/und hämatogen entstehen und betreffen bevorzugt die Haut, die Lunge, Lymphknoten, Leber und Hirn sowie Knochen und Nebennieren [1].

Das Stadium IV des malignen Melanoms ist nach der TNM-Klassifikation folgendermaßen definiert: „Jede Dicke des Primärtumors und jede Form der Lymphknotenmetastasierung in Kombination mit dem Auftreten von Fernmetastasen“. Die Prognose im Stadium IV des malignen Melanoms ist kritisch, der Tumor führt in der Regel schnell zum Exitus letalis. Wichtigste therapeutische Option ist die radikale Exzision des Primärtumors sowie eventuell vorliegender Metastasen. Inoperable Metastasen oder Resttumoren nach

\footnotetext{
${ }^{1}$ Die vorliegende Arbeit wurde als bestes klinisches Poster auf der 44. DDG-Tagung in Dresden 2007 ausgezeichnet. Die Arbeit wurde dem 10-jährigen Jubiläum der Berliner Stiftung für Dermatologie (BSD) gewidmet.
}

ben wird. Unsere Befunde zeigen, dass ein langfristiges Überleben von Melanom-Patienten im Stadium IV möglich ist. Entscheidend für die Prognose dieser Kranken sind die frühzeitige Erkennung der Metastasen und der rechtzeitige Einsatz sowohl operativer als auch pharmakologischer und strahlentherapeutischer Behandlungsmaßnahmen.

R1/R2-Resektion können stereotaktisch bestrahlt werden, bei Lebermetastasierung ist beispielsweise eine CT-gesteuerte Chemoembolisation eine Therapieoption. Eine adjuvante Immuntherapie mit Interferon kommt häufig zur Anwendung. Als Chemotherapeutika werden bei Fernmetastasierung insbesondere Dacarbazin, Temozolomid (hirngängiges Vorläufer-Medikament des Dacarbazins), die Kombination Carboplatin und Paclitaxel sowie Vindesine eingesetzt [2]. Trotz vielfältiger Therapieoptionen ist die Prognose im Stadium IV schlecht und die Therapie hat vor allem palliativen Charakter, d.h. sie soll die Beschwerden mindern und allenfalls die Überlebenszeit verlängern.

In dieser Studie werden einige Kranke aus der Hautklinik Minden beschrieben, die maligne Melanome mit Fernmetastasen hatten und mit verschiedenen, auf die jeweiligen Patienten zugeschnittenen Therapieschemata behandelt wurden. Es handelte sich um 11 Kranke, die innerhalb der letzten 10 Jahre wegen eines metastasierten Melanoms mit unterschiedlichen Therapieprotokollen von uns langfristig betreut wurden. In einer retrospektiven Aufarbeitung wurden diese elf Krankheitsverläufe mit den Daten der AJCC 2001 bezüglich des mittleren Überlebens von Patienten mit malignem Melanom im Stadium IV ( $\bullet$ Abb. 1 ) verglichen. 


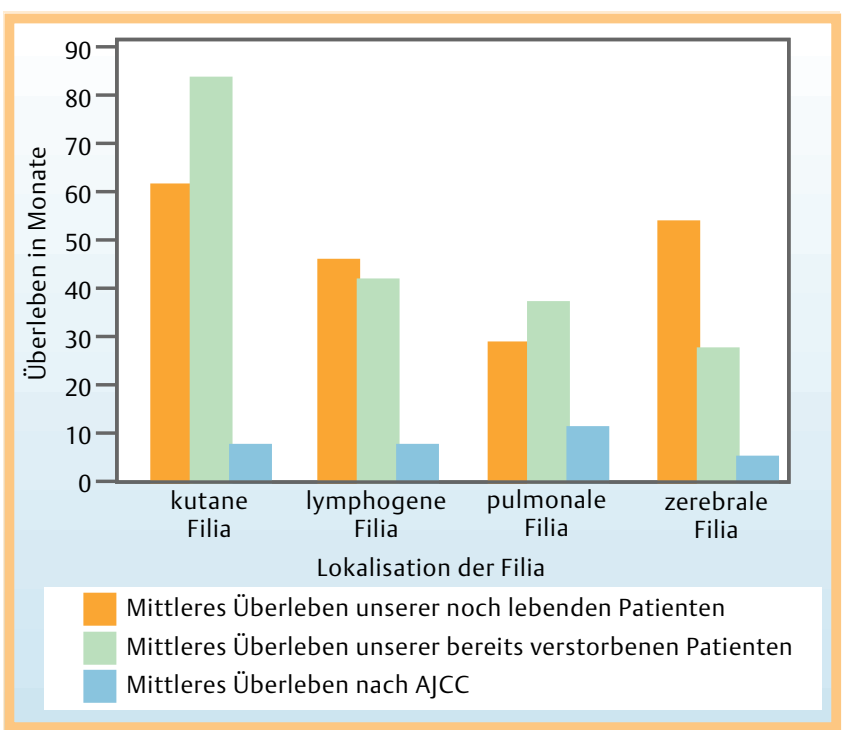

Abb. 1 Vergleich mittleres Überleben Hautklinik Minden vs. AJCC.

\section{Ergebnisse$$
\nabla
$$

Die wichtigsten Daten der 11 ausgewählten Patienten mit malignem Melanom im Stadium IV sind in $\bullet$ Tab. 1 zusammengefasst. Es handelt sich um 7 männliche und 4 weibliche Patienten, die bei Erstdiagnose 30 bis 65 Jahre alt waren (durchschnittlich 52,8 Jahre) und bei denen 3 primär-noduläre Melanome, ein ulzeriertes NM, 2 amelanotische NM und ein amelanotisches malignes Melanom sowie ein superfiziell spreitendes malignes Melanom, ein Schleimhautmelanom des Gaumens, ein BallonzellMelanom und ein nicht spezifizierbares malignes Melanom vorlagen. Die Tumordicke lag zwischen 1,1 und $10 \mathrm{~mm}$ und betrug im Durchschnitt 3,0 mm.

Der Primärtumor war in je drei Fällen an den unteren Extremitäten (Oberschenkel links, Knie rechts, Unterschenkel rechts) und am Stamm (Schulter links, Scapula rechts, Rücken) lokalisiert. Das Capillitium war zweimal betroffen, jeweils einmal die obere Extremität (Oberarm rechts) und die Mundschleimhaut (Gaumen). Bei einem Probanden blieb die Lokalisation des Primärtumors unbekannt. Die Metastasierung war bei 6 Patienten lymphogen, bei 6 Probanden fanden sich kutane Metastasen, bei 9 Patienten pulmonale, in sieben Fällen zerebrale und in einem Fall Lebermetastasen.

Alle Patienten wurden radikal operiert, d.h. Exzision des Primärtumors (sofern bekannt) sowie Exzisionen von einzelnen Metastasen (kutane, subkutane, pulmonale, zerebrale und Lymphknoten-Filiae) mit entsprechendem Sicherheitsabstand. Ferner erhielten alle Patienten eine Immuntherapie mit Interferon sowie ein bis drei Chemotherapien (DTIC, Temozolomid, Eldesine, Carboplatin und Paclitaxel u.a.). Primär wurde DTIC oder Temodal als Standard-Chemotherapie eingesetzt. Eine zusätzliche Bestrahlung erfolgte bei 4 Patienten.

Bei der Auswertung der Patientendaten wurde ein Gesamtüberleben von 38 - 149 Monaten (im Mittel 91,27 Monate) nach Erstdiagnose ermittelt.

Das mittlere Überleben unseres Patientenkollektivs mit Lymphknoten-Metastasen betrug 43,5 Monate ( 9 - 115 Monate), bei kutaner Metastasierung 72,83 Monate (30-115 Monate), nach pulmonaler Metastasierung überlebten die Patienten im Mittel 33,44 Monate (2-99 Monate), und nach zerebraler Metastasie-

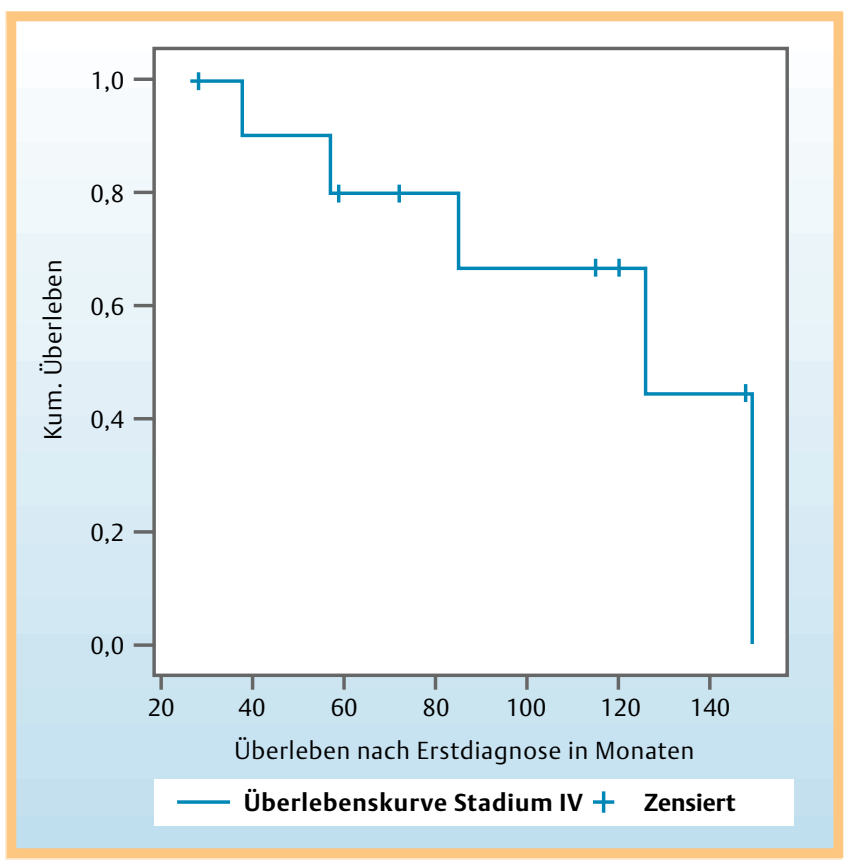

Abb. 2 Überleben nach Erstdiagnose im Stadium IV.

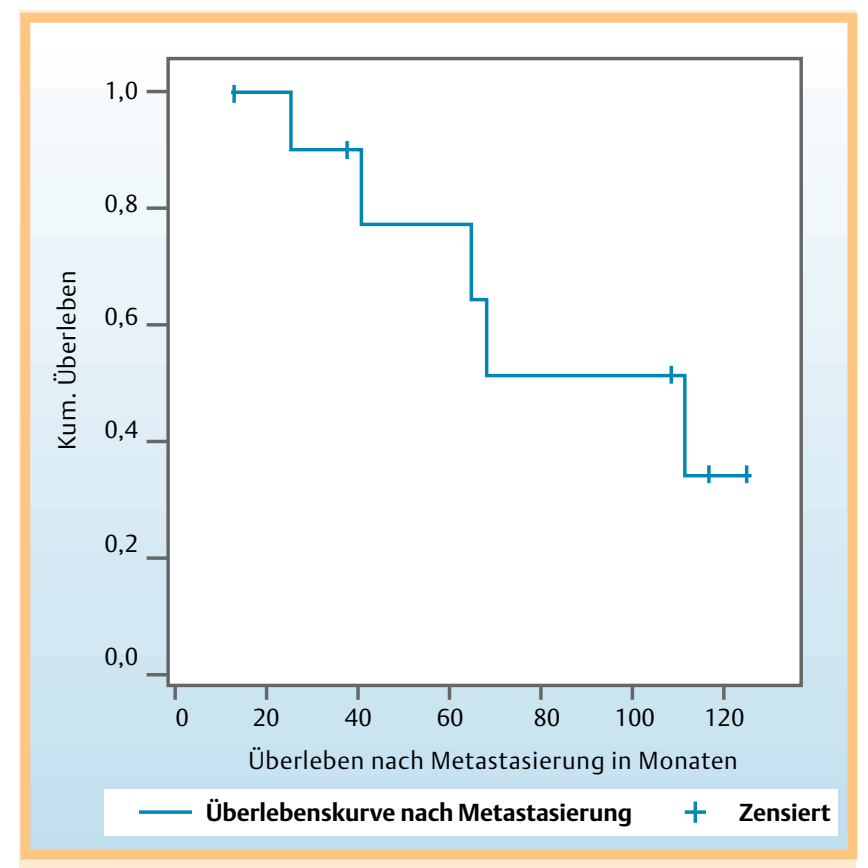

Abb. 3 Überleben nach Metastasierung im Stadium IV.

rung zeigte sich ein mittleres Überleben von 35 Monaten (2 - 115 Monate). Die eine Patientin mit Lebermetastasen überlebte nach Diagnosestellung fast 8 Monate.

Überlebenskurven nach Kaplan-Meier bezogen auf die Erstdiagnose und nach Feststellung einer Metastasierung finden sich in den 1 Abb. 2 und 3. 
(

It: $1: 32$

ตे

$\sigma \bar{m} \sigma \sim$

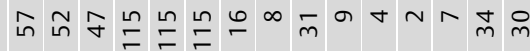

$\stackrel{\operatorname{m}}{\mathrm{N}} \stackrel{\infty}{\sim}$

$\stackrel{\ln }{\sim} 8$

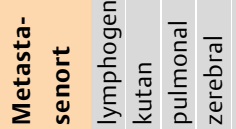

$\frac{\bar{\pi}}{\frac{\pi}{0}}$

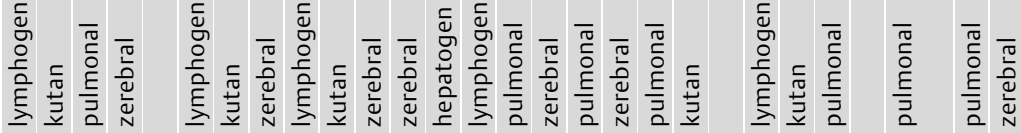

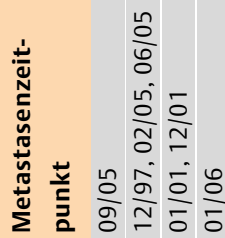

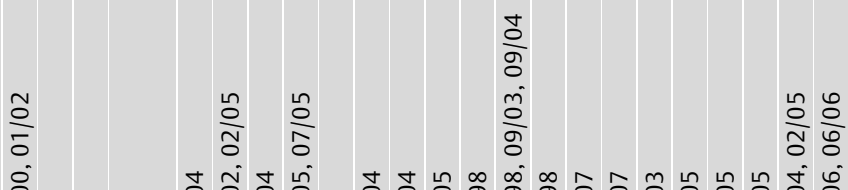

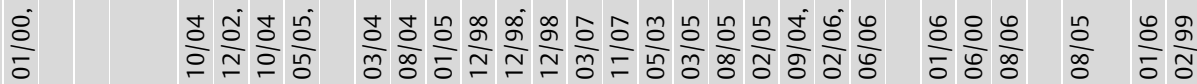

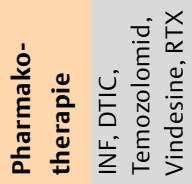

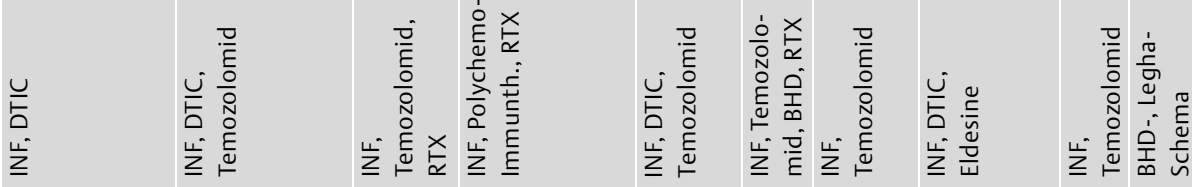

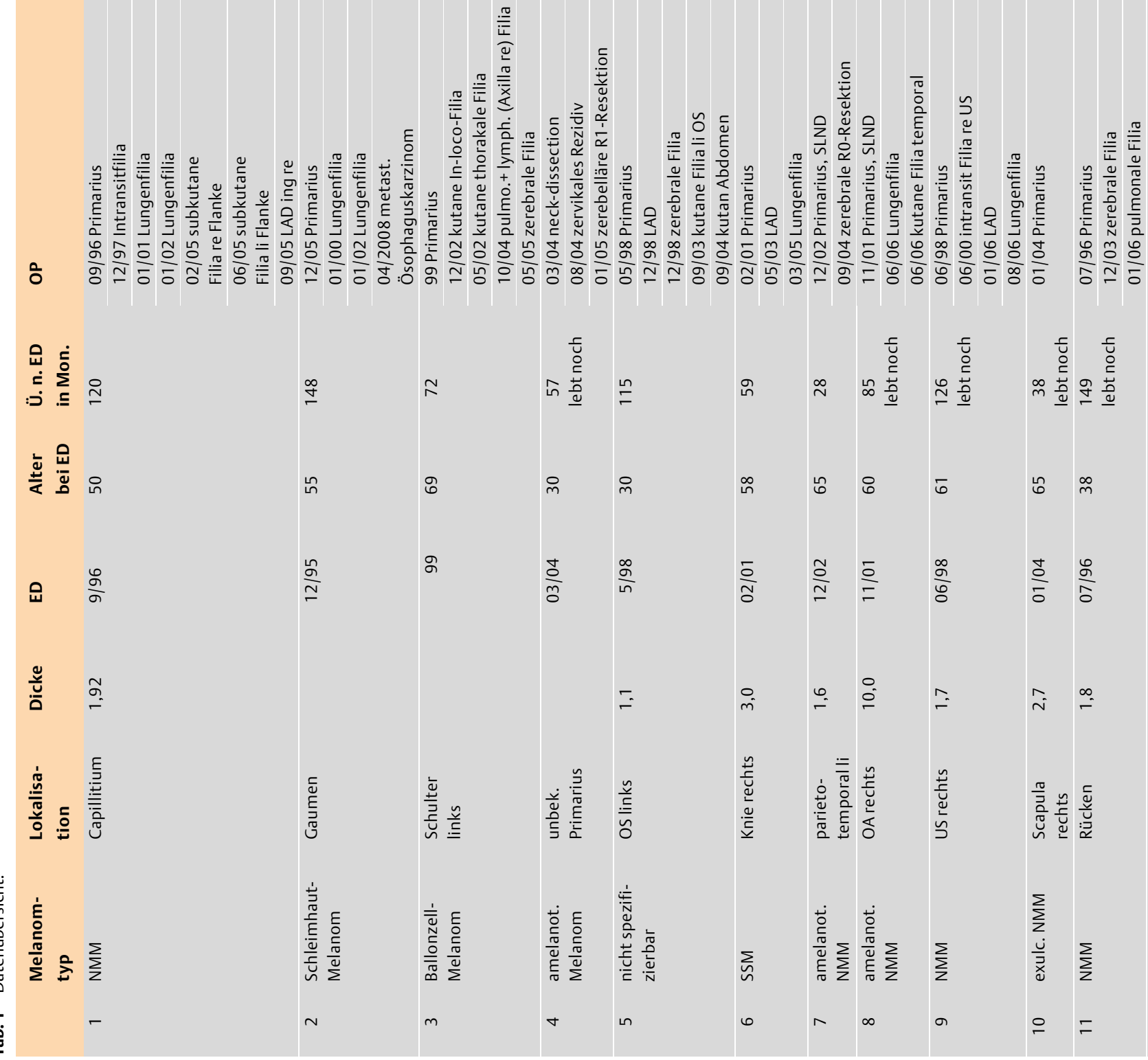




\section{Diskussion \\ $\nabla$}

Das mittlere Überleben von Patienten mit kutanen und Lymphknoten-Metastasen soll nach der AJCC 2001 7,2 Monate betragen, Patienten mit Lungen-Metastasen überleben im Mittel 11,4 Monate, Patienten mit zerebralen Metastasen 5,0 Monate. Eine vergleichende Darstellung dieser mit unseren Daten wird in - Abb. 1 gezeigt. Wir weisen darauf hin, dass hier Einzelfälle präsentiert werden und unser Patientenkollektiv nicht alle von uns behandelten Patienten einschließt.

$\mathrm{Zu}$ diskutieren bleibt, aus welchem Grund gerade die hier beschriebenen Patienten überdurchschnittlich lange überlebten. Eine Interferontherapie wurde in 10 von 11 Fällen eingesetzt (90,9\%), wobei bei allen Patienten ein schrittweises Vorgehen gewählt wurde: Zunächst wurde die Metastasenchirurgie eingesetzt und erst in zweiter Linie eine Chemo- oder Radiotherapie durchgeführt, wenn eine Operation nicht möglich war, oder als Ergänzung zu den chirurgischen Maßnahmen. Auch wenn die Leitlinie für Melanome im Stadium I und II eine bildgebende Diagnostik nicht zwingend empfiehlt, setzen wir zum Staging unserer Patienten frühzeitig derartige diagnostische Verfahren ein wie CT (Hals-Becken) und MRT(Hirnschädel) [4]. In neuester Zeit, wovon jedoch die hier dargestellten Patienten noch nicht profitieren konnten, kommt auch vermehrt die PET-CT bei Hochrisikopatienten oder unklarer CT/MRT zum Einsatz. Der hierdurch bedingte frühe Nachweis von Metastasen mit der Möglichkeit zum kurativen Therapieansatz stellt möglicherweise einen Überlebensvorteil für die Kranken dar, im Vergleich zu den Daten der AJCC.

Ein guter Ansatz, das Überleben von inoperablen Patienten im Stadium IV weiter zu verlängern, könnte die Chemosensitivitätstestung sein [5]. In einer Phase-II-Studie konnte zwischen chemosensiblen und chemoresistenten Patienten ein Unterschied festgestellt werden, wobei bei den chemosensiblen ein deutlich verlängertes Gesamtüberleben vorlag (14,6 vs. 7,4 Monaten). Auch konnte in einigen Fällen gezeigt werden, dass eine Mutationsanalyse und der gezielte Einsatz von Kinase-Inhibitoren zu einer Stabilisierung der Tumorkrankheit im Stadium IV führen kann [6]. Vermutlich wird es in Zukunft eine Kombination aus verschiedenen therapeutischen Möglichkeiten sein, die zwecks langfristiger Stabilisierung des Melanoms im Stadium IV eingesetzt werden. Ein solitärer Weg wird vermutlich nicht ausreichen.

Um das therapeutische chirurgische Vorgehen im Stadium III bei Vorliegen einer Mikro- oder Makrometastasierung prospektiv zu untersuchen und zukünftig die Entscheidung für oder gegen eine komplette Lymphadenektomie objektiv zu treffen, wird derzeit eine von der deutschen Krebshilfe finanzierte Wächterlymphknotenstudie unter der Leitung der Hautkliniken Tübingen und Minden durchgeführt.

Nach dem Einsatz neuer, heute schon zur Verfügung stehender diagnostischer und therapeutischer Möglichkeiten und der Auswertung weiterer Studien, werden in Zukunft die Überlebensdaten von Patienten mit malignem Melanom im Stadium IV neu bewertet werden müssen.

\section{Abstract}

\section{Long-Term Survivors With Malignant Melanoma Stage IV \\ $\nabla$}

The malignant melanoma is a highly malignant melanocytic tumour. Survival is short-timed and its final outcome in Stadium IV is always lethal. We present here 11 patients treated in our department for a longer period of time. Their overall survival was significantly longer, compared to the data given by the AJCC 2001.

Our observations suggest that long-term survival is possible in patients with stage IV malignant melanoma. The prognosis in progressed stages of melanoma depend on early recognition of the metastases, and, subsequently, the timely application of operative, pharmacological and radio-therapeutic measures.

\section{Literatur}

1 Orfanos CE, Garbe C (Hrsg). Therapie der Hautkrankheiten. 2. Aufl. Heidelberg: Springer, 2002

2 Garbe C. Management des Melanoms. Heidelberg: Springer, 2006

3 Balch CM et al. A multifactorial analysis of melanoma IV. Prognostic factors in 200 melanoma patients with distant metastases. J Clin Oncol 2001; 19: $3622-3634$

4 AWMF Leitlinie Malignes Melanom Nr. 032/024, Stand 09/2007

5 Ugurel $S$, Schadendorf $D$, Pföhler $C$ et al. In vitro drug sensitivity predicts response and survival after individualized sensitivity-directed chemotherapy in metastatic melanoma: a multicenter phase II trial of the Dermatologic Cooperative Oncology Group. Clin Cancer Res 2006; 12: $5258-5259$

6 Lutzky J, Bauer J, Bastian BC. Dose-dependent, complete response to imatinib of a metastatic mucosal melanoma with a K642E KIT mutation. Pigment Cell Melanoma Res 2008; 21: 492 -493. Epub 2008 May 29 